UNIVALI

UNIVERSIDADE DO VALE DO ITAJAI

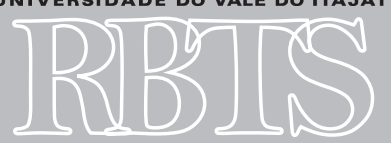

Revista Brasileira de Tecnologias Sociais
1 Ensaio apresentado no Congresso Internacional de Políticas Públicas (UNIVALI, Itajaí, 21 de junho de 2017), na mesa "Políticas Públicas e a Pesquisa na América Latina - olhares e caminhos". 2 Professor de Administração Pública - Universidade do Estado de Santa Catarina (UDESC) -leonardo.secchi@udesc.br 3 Professor de Administração Pública - Universidade do Estado de Santa Catarina (UDESC) -ricardo.cavalheiro@udesc.br 4. Diretora do think tank PVBLICA - leticia@pvblica.org.br

\section{OS THINK TANKS NA AMÉRICA LATINA E A PRODUÇÃO DE CONHECIMENTO APLICADO À POLÍTICA PÚBLICA ${ }^{1}$}

\section{LATIN AMERICAN THINK TANKS AND THE PROMOTION OF KNOWLEDGE APPLIED TO PUBLIC POLICIES}

\author{
Leonardo Secchi ${ }^{2}$ \\ Ricardo Alves Cavalheiro ${ }^{3}$ \\ Letícia Elena Ito ${ }^{4}$
}

RESUMO: Este ensaio traz ao debate o papel e a relevância da produção de conhecimento aplicado em políticas públicas, dando ênfase aos think tanks. Para tanto, faz-se uma discussão inicial dos conceitos de políticas públicas, problema público e produção de conhecimento aplicado como uma das características fundamentais da área de análise de políticas públicas. Os think tanks surgem como instituições especializadas neste papel. Na América Latina existem 695 think tanks que utilizam métodos e técnicas da policy analysis e da policy advocacy para produzir conhecimento aplicado e influenciar os processos decisórios, de implementação e avaliação de políticas públicas.

PALAVRAS-CHAVE: Política pública; problema público; conhecimento aplicado.

ABSTRACT: This essay discusses the role and relevance of the production of applied knowledge in public policies, focusing specifically on think tanks. It initially discusses the concepts of public policies, public problem and the production of applied knowledge as one of the fundamental characteristics of the area of analysis of public policies. Think tanks emerge as specialized institutions in this role. In Latin America there are 695 think tanks that use methods and techniques of policy analysis and policy advocacy to produce applied knowledge and influence the decision-making, implementation and evaluation processes in regard to public policies.

KEYWORDS: Public policy; public problem; applied knowledge. 
UNIVALI

UNIVERSIDADE DO VALE DO ITAJAÍ

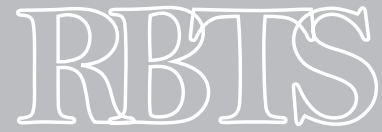

Revista Brasileira de Tecnologias Sociais 


\section{INTRODUÇÃO}

A política pública pode ser entendida como a diretriz e sua ação coordenada ao enfrentamento de um problema público. A tradução prática da política pública resulta em diretriz voltada à transformação positiva da realidade pública. Nesse sentido, mostra-se oportuno esclarecer que o termo "política” (policy) não se confunde com conteúdo presente no jargão popular sobre “política” (politics), notadamente vinculado ao exercício e à manutenção do poder pelo e para os atores políticos (BOBBIO, 2002). Nessa seara, o vocabulário internacional, em especial da língua inglesa, permite uma melhor compreensão, visto que o termo "politics" se refere à prática política, enquanto o "policy” à produção de diretrizes de enfrentamento de qualquer problema público.

Outro ponto relevante que se tem no entendimento de políticas públicas reside na delimitação do problema público. Diante disso, mostra-se oportuno destacar que um problema é alçado à condição de público quando uma quantidade e qualidade significativa dos atores sociais se consideram presentes em uma situação indesejada e vislumbram uma possibilidade real e viável de alterar positivamente a realidade pública, seja na área da saúde, educação, segurança, mobilidade, meio ambiente (SJÖBLOM, 1984; SECCHI, 2013).

A quantidade é fundamental para diferenciar problemas particulares dos problemas da coletividade. Já a qualidade é relevante, pois atores em destaque, os mass media, instituições de classes, grupos de interesses, entres outros, possuem maior capacidade de disseminação de ideias, projetos e mobilização de interessados. Com isso, vê-se que, a depender da qualidade dos atores envolvidos, há uma maior chance de que situações identificadas como indesejadas entre nas pautas de discussões públicas, enquanto outras, ainda que impróprias ao bem-estar coletivo, continuem abaixo do radar da agenda pública. A natureza do surgimento do problema também pode influir na opinião pública, visto que ele pode se dar de forma súbita, como nos casos de epidemia e ondas de criminalidades; também pode se dar lentamente, a ponto de restar insuportável, como no caso de problemas de mobilidade urbana; ou ainda de forma sazonal, como nos problemas de secas e doenças relacionadas ao clima. Uma vez identificado como problema público, tem-se nas políticas públicas o meio técnico de se transformar a situação indesejada na realidade pretendida (SECCHI, 2013).

E quem comanda as ferramentas necessárias à construção da nova realidade? Quem possui o poder para tanto? Há quem defenda a visão estadocêntrica que afirma que somente ao governo estatal cabe o papel fazer política pública. Contudo se entende que, além da estrutura de governo, há múltiplos atores que compõem e atuam na arena pública nas diversas etapas existentes no planejamento, na execução e na avaliação de políticas públicas, tais como: os policymakers, policytakers, os analistas de políticas públicas, a mídia, etc. Por isso, a perspectiva de políticas públicas a partir de uma visão multicêntrica é evidentemente mais abrangente do que a de políticas governamentais. Soma-se a isso, ainda, a visão de que a ação é elemento estruturante das políticas públicas e nunca a omissão. Com isso, vê-se que a decisão de não agir diante de um problema público não pode ser analisada como uma formulação de política pública. E tal condição independe de se tratar em ato em nível micro ou macro, pois se entende que a política pública sempre poderá ocorrer nos diversos níveis de interação institucional (DYE, 1972; HOWLETT; RAMESH; PERL, 2013; DROR, 1971; KOOIMAN, 1993; RHODES, 1996; REGONINI, 2001; HAJER, 2003; GIULIANI, 2005; SECCHI; 2013).

Diante da diversidade de etapas no processo de elaboração de políticas públicas, assim como das peculiaridades envolvidas em cada um dos momentos, resta evidente a importância da 
produção de conhecimento analítico aplicado, pois, pela análise apurada, é possível identificar o papel dos atores na identificação e na promoção à inserção da agenda pública problemas reais ou retóricos, assim como se a pauta pública está relacionada verdadeiramente com o interesse coletivo, assim como se o conteúdo é eminentemente técnico ou político. Além disso, o estudo sistematizado oferece ferramentas de análises que permite a identificação da qualidade das propostas de enfrentamento público, assim como a parametrização de políticas reais, simbólicas, pseudopolíticas ou políticas sem sentido diante do conhecimento disponível para a elaboração e implementação da política pública e a intenção genuína do gestor de alterar a realidade pública (LOWI, 1972; WILSON, 1983; GORMLEY, 1986; GUSTAFSSON, 1983, BOZEMAN; PANDEY, 2004).

A partir disso, a produção de conhecimento especializado em política pública depende de técnicas e métodos já experimentados, seja pela abordagem racionalista (WEIMER; VINING, 1998) ou pela abordagem argumentativa (FISCHER; FORESTER, 1993). Nessa seara, a análise prescritiva de políticas públicas abarca os aspectos legais, sociais e econômicos que são imperiosos para embasar a tomada de decisão, bem como para apoiar a implementação e traçar quadros de avaliação sobre o sucesso e a efetividade do emprego de recursos públicos. Trata-se de área de intersecção de diversos campos técnicos de conhecimento, tais como: direito, economia, finanças, engenharia, urbanismo, contabilidade, sociologia. Faz-se necessária a tradução dos termos técnicos em informações compreensíveis a toda população como financiadora e destinatária final das políticas públicas (HEIDEMANN; SALM, 2009; HOWLETT; RAMESH; PERL, 2013; SECCHI, 2013).

Vê-se, portanto, que o exercício da atividade pública não pode ser lançado à sorte da aleatoriedade das realidades locais ou ainda ser refém exclusivo do conhecimento popular. Pelo contrário, deve ser amparado por robusta produção de métodos, sistemas e parâmetros espelhados nas melhores práticas do campo do conhecimento das políticas públicas capazes de integrar transversalmente os variados campos do saber.

Precisamente disso se dedica a ciência da política pública (policy sciences), principalmente a partir da década de 50. Considera-se a pedra fundamental deste campo de conhecimento a obra "The Policy Sciences” de David Lerner e Harold Lasswell. A referida ciência visa à análise multidisciplinar teórica e prática das relações entre governo, instituições e cidadãos, assim como a construção de conhecimento útil descritivo e prescritivo destinados à resolução de problemas atinentes aos diversos setores de intervenção pública (HEIDEMANN; SALM, 2009; SECCHI, 2016).

A atividade de análise de políticas públicas (policy analysis) tem sua origem numa atividade prática: sistematizar processos, procedimento e informações com o objetivo de apoiar o processo decisório de políticas públicas e prescrever as melhores alternativas técnicas de resolução do problema público. Dados estatísticos, financeiros, econômicos, políticos e tecnológicos são dissecados pelos analistas de política pública e transformados em soluções práticas que satisfaçam às demandas do gestor público de acordo com a dinamicidade, velocidade e urgência de cada realidade pública. Sendo assim, os métodos de trabalho utilizados pelos analistas de política pública se aproximam da linguagem técnica vivida pelo gestor público, assim como o resultado se vê em relatórios prescritivos de apoio à tomada de decisão e não em livros teóricos ou artigos científicos, tal como ocorre no campo da ciência da política pública. 
As principais organizações especializadas na produção de conhecimento aplicado em política pública são os think tanks (SOARES, 2009; SECCHI, 2016).

De acordo com a literatura norte-americana, os think tanks são entidades independentes que realizam pesquisas e produzem conhecimento significativo na área de políticas públicas. Além disso, preenchem uma lacuna que há entre a academia e o governo, visto que os think tanks inseremse entre o mundo das ideias e o mundo da ação (HAASS, 2002). Eles surgiram nos Estados Unidos da América (EUA) no início do século XX e hoje já soam 6.846 think tanks em todo o mundo (MCGANN, 2016), fruto da progressiva percepção que não é possível fazer política pública sem ter base técnica e argumentativa sólida para a sua elaboração.

Existem diferentes tipos de think tanks, variam tanto na área de atuação quanto na própria estrutura institucional. No que tange à atuação, alguns são voltados para produção e propagação de conhecimento, outros para análise de políticas públicas e, ainda, algumas dessas instituições realizam policy advocacy para que determinados assuntos entrem na agenda política e institucional, e formação da opinião pública (STONE, 2007; SECCHI, 2013; MCGANN, 2016).

Quanto à estrutura, há think tanks que utilizam a estrutura formal do governo, outros que se vinculam a empresas privadas (até compartilham a finalidade de obtenção de lucro), a partidos políticos, a confederações. Mas grande parte deles são institutos independentes sem fins lucrativos (MCGANN, 2016).

Destaca-se que os think tanks voltados para a elaboração e divulgação de conhecimento o fazem instrumentalizando os problemas reais, visto que eles normalmente são acionados para resolver problemas já existentes, diferentemente de uma universidade que, predominantemente, investe seus esforços em elaborar e aprofundar teorias (SECCHI; ITO, 2016). Além disso, os think tanks possuem caráter inovador, buscam novas soluções para os problemas. Tal característica os diferencia de uma consultoria que utiliza conhecimentos previamente estabelecidos (SECCHI, 2013).

Apesar da propagação dos think tanks em nível mundial, de acordo com Albornoz, Correa e Darraz (2014), o termo think tank propriamente dito não foi demasiadamente incorporado na América Latina, as entidades são mais conhecidas como centro de investigação, consultoria, centro de pensamento, organizações não governamentais (ONG) ou associação civil. Além disso, conforme Botto (2011), os think tanks em muitos países da América Latina foram criados para contrapor os regimes autoritários, pois a sociedade clamava por seus direitos e liberdade, diferentemente dos EUA e outros países que já tinham essas questões de natureza social mais consolidada. O Brasil é uma exceção, conforme Secchi e Ito (2016). Os primeiros think tanks surgiram na época da ditadura, mas com o intuito de auxiliar na resolução dos problemas públicos e também, de certa forma, contribuíam com o fortalecimento do governo autoritário.

Com o passar do tempo, de acordo com Botto (2011), os governos autoritários foram se extinguindo na América Latina e novos problemas foram surgindo, consequentemente, novos think tanks. As organizações de origem eram sindicatos, governo, empresas, entre outros; todavia se destaca que a grande maioria é derivação de universidades e terceiro setor. E, ao contrário dos think tanks norte-americanos, que se caracterizam por sua independência na sua organização de origem, os think tanks latino-americanos sofrem bastante influência dessas organizações em seu modus operandi. 
Por exemplo, os think tanks que derivam de universidades possuem muitos cientistas em seu quadro de pessoal e realizam pesquisas longas, cujos resultados são quadros analíticos, e tais resultados são enviados para serem publicados em revistas acadêmicas (BOTTO, 2011).

Em 2008, havia 481 think tanks latino-americanos, já em 2016 há registro de 695 (MCGANN, 2008, 2016). Esses últimos dados são demonstrados no Gráfico 01.

Gráfico 1: Número de think tanks na América Latina

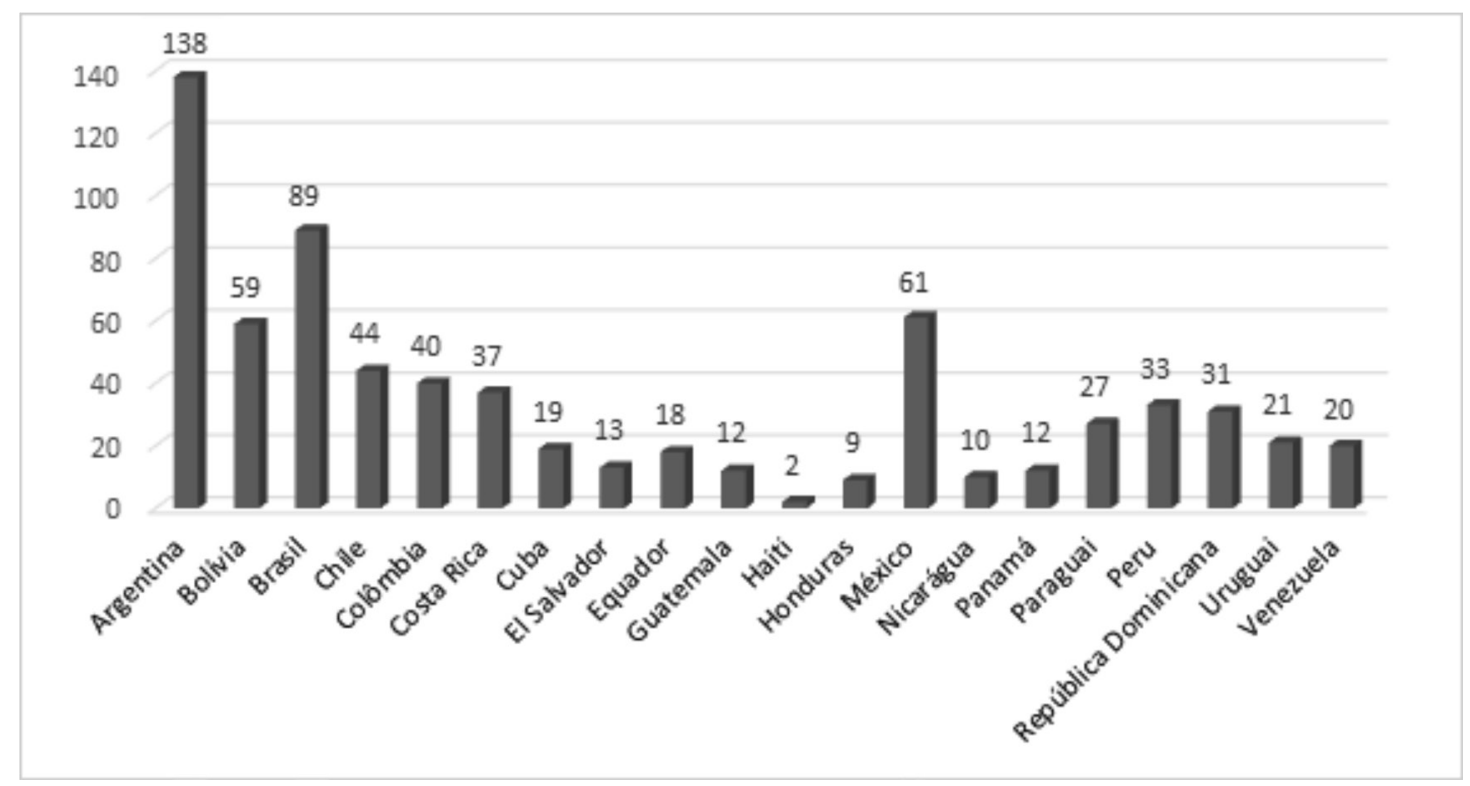

Fonte: Global Go To Think Tank Index Report, de McGann (2016).

Destacam-se, entre os think tanks mais relevantes da América Central e América do Sul, os seguintes think tanks, segundo ranking elaborado pela Universidade da Pensilvânia (McGANN, 2016):

1. Fundação Getúlio Vargas - FGV (Brasil - fundação privada sem fins lucrativos que faz atividades de ensino, pesquisa aplicada e consultoria).

2. Comisión Económica para América Latina - CEPAL (Chile - instituição ligada ao sistema das Nações Unidas voltada para estudos aplicados de políticas públicas para o desenvolvimento econômico da América Latina).

3. Fedesarollo (Colômbia - instituição privada sem fins lucrativos voltada para a formulação, monitoramento e aprimoramento das políticas públicas de desenvolvimento econômico e social da Colômbia).

Outros think tanks de destaque na América Latina são o Centro Brasileiro de Relações Internacionais (CEBRI), o Instituto de Pesquisa Econômica Aplicada (IPEA), o Consejo Latinoamericano de Ciencias Sociales (CLACSO), Centro Brasileiro de Análise e Planejamento (CEBRAP) e a Fundación para el Avance de las Reformas y las Oportunidades (Grupo FARO).

O aumento do número de think tanks, de acordo com Garcé e López (2014), se deu por três razões na região latino-americana. A primeira ocorreu na época dos regimes autoritários, em alguns dos países, quando professores foram excluídos das universidades públicas e fundaram instituições para trabalhar no ramo da consultoria. Já a segunda razão foi num período em que as universidades tinham que priorizar a docência, pois o governo assim queria; por consequência, as pessoas que tinham interesse em desenvolver pesquisas orientadas na área de políticas públicas 
também recorreram às instituições. Por fim, a terceira razão foi nos anos 1990 em que houve uma mudança de perspectiva, mais voltada para o mercado e, ainda, o Estado foi perdendo sua centralidade e isso fez com que a sociedade civil se fortalecesse, bem como suas organizações.

A produção de conhecimento, no território latino-americano, continua sendo primordialmente realizada pelas universidades, porém, ao longo dos anos, os think tanks vêm conquistando espaço, especialmente no que tange ao conhecimento em ciência social aplicada, orientado ao cliente (governos, $\mathrm{ONG}^{\prime}$, , confederações, etc.), com novos métodos para sua produção e disseminação (BOTTO, 2011). Entretanto, as universidades são mais reconhecidas como produtoras de conhecimento do que os think tanks (ALBORNOZ; CORREA; DARRAZ, 2014; SECCHI; ITO, 2014). Mesmo havendo diferenças entre essas organizações, elas contribuem para o campo das ciências sociais, que englobam: administração pública, ciência política, relações internacionais, sociologia, educação, transporte, estudos urbanos, economia, econometria e finanças (BRUNNER et al., 2014).

Inclusive, de acordo com Brunner et al. (2014), não há estudos aprofundados sobre os resultados dos think tanks de forma isolada. Assim sendo, a produção técnico-científico é mensurada sem segregar a produtividade dos think tanks e das universidades. No Quadro 1, apresenta-se, de forma sucinta, a produção no âmbito das ciências sociais.

Quadro 1: Produção das ciências sociais na América Latina

\begin{tabular}{|l|c|c|c|}
\hline Indicador (América Latina) & Quantidade & Ano & Fonte \\
\hline $\mathrm{N}^{\mathrm{o}}$ de think tanks & 695 & 2016 & McGann (2016) \\
\hline $\mathrm{N}^{\mathrm{o}}$ de universidades (públicas e privadas) & $4.081^{*}$ & 2014 & $\begin{array}{l}\text { Brunner e Miranda } \\
\text { (2016) }\end{array}$ \\
\hline $\begin{array}{l}\text { Produção relevante na área de ciências sociais } \\
\text { (artigos publicados) }\end{array}$ & 19.356 & 2014 & Brunner et al. (2014) \\
\hline
\end{tabular}

Fonte: Elaboração dos autores.

Notas: * Não constam dados do Haiti.

Segundo Albornoz, Correa e Darraz (2014), os think tanks latino-americanos, de maneira geral, estão mais focados em influenciar a agenda pública do que dedicar atenção nas etapas da concepção ou implementação de políticas públicas. Inclusive, as relações com os governos são pontuais, ou seja, não há um envolvimento permanente e os governos recorrem aos think tanks normalmente em momentos de crise ou extrema gravidade.

Outro ponto importante que interfere nos think tanks latino-americanos é a questão dos recursos financeiros. Os países da América Latina não disponibilizam muitos de seus recursos para fomentar os think tanks, entretanto as organizações captam de duas formas os recursos públicos. Um deles quando fornecem serviço de consultoria para auxiliar na solução de problemas públicos junto ao governo. A outra forma é quando o governo disponibiliza recursos para fomentar áreas de ciência, tecnologia e inovação. Todavia, o grande aporte financeiro advém de cooperações internacionais, nesse caso, think tanks latino-americanos concorrem com mais de 6.500 think tanks existentes mundo afora (ALBORNOZ; CORREA; DARRAZ, 2014).

\section{CONSIDERAÇÕES FINAIS}

Por sua trajetória e legado histórico de dependência e subdesenvolvimento, os países da América Latina precisam elaborar suas políticas públicas com base técnica, idiossincrasia política 
e sensibilidade social. Em outras palavras, a cópia acrítica de políticas públicas vindas dos países centrais para resolver problemas da América Latina é um caminho enganoso.

Para dar conta das peculiaridades de cada país, região ou localidade, é necessária a especialização linguística, metodológica e argumentativa para a formulação e implementação de políticas públicas adequadas ao arcabouço institucional, cultural, legal e de atores políticos da própria América Latina.

Para tanto, a área de políticas públicas (policy sciences) e a atividade de análise de políticas públicas (policy analysis) são fundamentais para a formação de governantes, analistas, assessores, pesquisadores que tenham condições de diagnosticar problemas públicos nas diversas áreas (saúde, educação, previdência, etc.) e apontar recomendações de políticas públicas adequadas.

As universidades e os think tanks são fundamentais para a formação desta massa crítica. Os primeiros avançando o conhecimento teórico e metodológico sobre política pública (como campo de conhecimento) e sobre os temas de políticas públicas (conhecimento aplicado às áreas de intervenção governamental).

Já os think tanks, como demostrado no ensaio, são atores especializados na produção de conhecimento aplicado, voltado para o tomador de decisão, para auxiliá-lo a fazer política pública customizada às suas necessidades. É importante a percepção destes atores, seu fomento e sua utilização para que os países da América Latina possam desenvolver políticas públicas tecnicamente preparadas, socialmente justas e politicamente aceitáveis.

\section{REFERÊNCIAS}

ALbORnOZ, M.; CORREA, N.; DARRAZ, E. F. Introducción. In: RIED, J. J. B.; CEDEÑO, O. J. B.; ALBUJA, A. M. A. Más saber América Latina: potenciando el vínculo entre think tanks y universidades. Quito: Grupo Faro; CPCE, 2014.

BOBBIO, N. Política. In: BOBBIO, N.; MATEUCCI, N.; PASQUINO, G. Dicionário de Política. 12. ed. v.2. Brasília: UnB, 2002.

BOTTO, M. Think Tanks in America Latina: radiografia comparada de un nuevo actor político. In: ASTE, N. C.; MENDIZABAL, E. Vínculo entre conocimiento y política: el rol de la investigación en el debate público en America Latina. Peru: Consorcio de Investigación Económica y Social (CIES), 2011.

BOZEMAN, B.; PANDEY, S. K. Public management decision making: effects of decision content. Public Administration Review, v. 64, n. 5, p. 553-565, 2004.

BRUNNER, J. J.; BELLETTINI, O.; ARELLANO, A.; VILLALOBOS, C. Conclusiones y recomendaciones. In: RIED, J. J. B.; CEDEÑO, O. J. B.; ALBUJA, A. M. A. Más saber América Latina: potenciando el vínculo entre think tanks y universidades. Quito: Grupo Faro; CPCE, 2014.

BRUNNER, J. J.; MIRANDA, D. A. Educación superior en iberoamérica: informe 2016. Chile: CINDA, 2016.

DROR, Y. Design for policy sciences. New York: American Elsevier Publishing Company, 1971.

FISCHER, F.; FORESTER, J. (Org.). The argumentative turn in policy analysis and planning. Durham: Duke University Press, 1993.

GARCÉ, A.; LÓPEZ, C. Estudio Regional. In: RIED, J. J. B.; CEDEÑO, O. J. B.; ALBUJA, A. M. A. Más 
saber América Latina: potenciando el vínculo entre think tanks y universidades. Quito: Grupo Faro; CPCE, 2014.

GIULIANI, M. Policy Termination. In: CAPANO, G.; GIULIANI, M. (Ed.). Dizionario di politiche pubbliche. Roma: Carocci, 2005.

GORMLEY Jr., W. T. Regulatory issue networks in a federal system. Polity, v. 18, n. 4, p. 595-620, 1986.

GUSTAFSSON, G. Symbolic and pseudo policies as responses to diffusion of power. Policy sciences, v. 15, n. 3, p. 269-287, 1983.

HAJER, M. Policy without polity? Policy analysis and the institutional void. Policy sciences, v. 36, n. 2, p. 175-195, 2003.

HAASS, R. N. Think Tanks and U.S. Foreign Police: a policy-maker's perspective. Estados Unidos da América: Department of State, 2002. Disponível em: <https://2001-2009.state.gov/s/p/rem/15506. htm>. Acesso em: 12 jun. 2017.

HEIDEMANN, F. G.; SALM, J. F. (Org.), Políticas públicas e desenvolvimento. Brasília: UnB, 2009.

HOWLetT, M.; RAMESH, M.; PERL, A. Política Pública: seus ciclos e subsistemas, uma abordagem integral. Rio de Janeiro: Elsevier, 2013.

KOOIMAN, J. (Ed.). Modern governance: new government-society interactions. Sage, 1993.

LOWI, T. J. Four systems of policy, politics, and choice. Public administration review, v. 32, n. 4, p. 298-310, 1972.

MCGAnN, J. G. 2008 Global Go To Think Tank Index Report. Pennsylvania: University of Pennsylvania Press, 2008.

MCGAnN, J. G. 2012 Global Go To Think Tank Index Report. Pennsylvania: University of Pennsylvania Press, 2012.

MCGAnN, J. G. 2016 Global Go To Think Tank Index Report. Pennsylvania: University of Pennsylvania Press, 2016.

REGONINI, G. Capire le politiche pubbliche. Il Mulino, 2001.

RHODES, R. A. W. The new governance: governing without government. Political studies, v. 44, n. 4, p. 652-667, 1996.

SECCHI, L. Políticas Públicas: conceitos, esquema de análise, casos práticos. 2. ed. São Paulo: Cengage Learning, 2013.

SECCHI, L. Análise de políticas públicas: diagnóstico de problemas, recomendação de soluções. São Paulo: Cengage Learning, 2016.

SECCHI, L.; ITO, L. E. Brasil. In: RIED, J. J. B.; CEDEÑO, O. J. B.; ALBUJA, A. M. A. Más saber América Latina: potenciando el vínculo entre think tanks y universidades. Quito: Grupo Faro; CPCE, 2014.

SECCHI, L.; ITO, L. E. Think tanks e universidades no Brasil: análise das relações na produção de conhecimento em política pública. In: Planejamento e políticas públicas. n.46, Jan/Jun. Brasília: Ipea, 2016. 
SJÖBLOM, G. Problemi e soluzioni in politica. Rivista Italiana di Scienza Politica, v. 14, n. 01, p. 4185,1984 .

SOARES, J. R. Think tanks: organização sistêmica de conhecimentos relevantes a política pública no Brasil. 2009. Dissertação (Mestrado Profissional em Administração), Universidade do Estado de Santa Catarina, Florianópolis. 2009.

STONE, D. Recycling bins, garbage cans or think tanks? Three myths regarding policy analysis institutes. United Kingdom: University of Warwick Institutional Repository, 2007.

WEIMER, D. L., VINING, A. Policy analysis: concept and practice. Upper Saddle River, NJ.: Prentice Hall, 1998.

WILSON, J. Q. American government: institutions and policies. Lexington: Heath \& Co., 1983. 